\title{
Estimación de biomasa y contenido de carbono de Pinus cooperi Blanco, en Pueblo Nuevo, Durango
}

\author{
Dorian de J. Pimienta de la Torre ${ }^{1}$, Gabriel Domínguez Cabrera ${ }^{1}$, \\ Óscar Aguirre Calderón ${ }^{1}$, Francisco Javier Hernández², \\ y Javier Jiménez Pérez ${ }^{1}$
}

\section{RESUMEN}

El objetivo del presente trabajo fue la estimación de la biomasa y del carbono contenido en bosques de Pinus cooperi Blanco, en el ejido La Victoria, Pueblo Nuevo, Durango. Empleando datos provenientes de ocho parcelas de muestreo de $5000 \mathrm{~m}^{2}$ y 633 árboles muestreados, se realizó el proceso matemático, encontrando que el modelo que presenta un mejor ajuste para la variable biomasa seca es $B=22,3476+(-4,9470)^{*} D+0,4911 * D^{2}+0,0039 *\left(D^{2} H\right)$, con un coeficiente de determinación $\left(R^{2}\right)$ de 0,99 y un error estándar porcentual $\left(S_{x} \%\right)$ de 11,69 . El modelo para la variable carbono contenido fue $C c=11,5090+(-3,1229) * D+0,3100 * D^{2}+0,0004 *\left(D^{2} H\right)$, el cual obtuvo un $R^{2}$ de 0.99 y un $S_{x} \%$ de 2,46 , respectivamente. Una vez determinado el mejor modelo se elaboraron tablas de estimación de biomasa y carbono contenido o almacenado para las diferentes categorías diamétricas y de altura.

PALABRAS CLAVE:

Biomasa, carbono, Durango, Pinus cooperi Blanco, volumen.

\begin{abstract}
The objective of the present research was to estimate the biomass and the carbon content in Pinus cooperi Blanco forests, in Pueblo Nuevo, Durango at La Victoria ejido. Using data originating from 8 sampling plots of $5000 \mathrm{~m}^{2}$ and 633 sample trees the mathematical process was carried out, finding that the model that presents a better adjustment for the biomass variable was $B=22,3476+$ $(-4,9470) * D+0,4911 * D^{2}+0,0039 *\left(D^{2} H\right)$, with a determination coefficient $\left(R^{2}\right)$ of 0,99 and a percentage standard error $\mathrm{S}_{x} \% 11,69$. The model for the carbon content variable was $\mathrm{Cc}_{\mathrm{c}}=11,5090+(-3,1229)$ * $D+0,3100$ * $D^{2}+0,0004$ * $\left(D^{2} H\right)$, which produced a $R^{2}=0,99$ and a $S_{x}=2,46$ respectively. Once the best model was determined the estimation tables for biomass and carbon content were ellaborated for the different diametric and height categories.

KEY WORDS:

Biomass, carbon, Durango, Pinus cooperi Blanco, volume.
\end{abstract}

1 Facultad de Ciencias Forestales, Universidad Autónoma de Nuevo León. Carr. Nacional km 145. Linares, Nuevo León, México. ce: djpimient@hotmail.com

2 Instituto Tecnológico de El Salto, Mesa del Tecnológico s/n, El Salto, Durango, México. 


\section{INTRODUCCIÓN}

Actualmente, se reconoce que el clima global se verá alterado significativamente en el presente siglo como resultado del aumento de concentraciones de gases invernadero, tales como el bióxido de carbono $\left(\mathrm{CO}_{2}\right)$, metano $\left(\mathrm{CH}_{4}\right)$, óxidos nitrosos $\left(\mathrm{NO}_{\mathrm{x}}\right)$ y clorofluorocarbonos (CFC's) (Ordóñez y Masera, 2001).

El incremento en las concentraciones de dichos gases en la atmósfera, debido a las actividades humanas, da origen al cambio climático (Ordóñez, 1999). Cada vez es más evidente que las emisiones de gases de efecto invernadero están afectando el clima del planeta, producto de la quema de combustibles fósiles y a cambios de uso de suelo, en particular a la deforestación (IPCC, 2001; Schlegel, 2001). Uno de los gases de efecto invernadero más importantes por la cantidad y velocidad en que se emite, es el $\mathrm{CO}_{2}$ (Ordóñez, 1999).

Los bosques juegan un papel importante en el ciclo del carbono global (Masera, 2001). Como sistema natural complejo contribuyen a mitigar el cambio climático global al almacenar carbono en la vegetación y en el suelo, e intercambiar carbono con la atmósfera a través de los procesos fotosintéticos y de respiración (Gasparri y Manghi, 2004). El proceso de fotosíntesis fija de forma natural el carbono, y a su vez mitiga el problema de cambio climático (Karjalainen, 1996).

La vegetación forestal absorbe $\mathrm{CO}_{2}$ durante su dinámica de crecimiento, es decir, fija el carbono para la composición de su estructura (tallos, ramas, hojas y raíces) y libera el oxígeno que proporciona un beneficio ambiental a todo el planeta. Los países (Anexo I) que demuestren una decidida política de conservación o incremento de sus superficies forestales, tendrán oportunidad de ingresar al mercado de la venta internacional de certificados de reducción de emisiones a través de los mecanismos de desarrollo limpio (SEMARNAT, 2001).

La captura de carbono atmosférico mediante prácticas de manejo del bosque, está en función de la acumulación y almacenamiento del mismo en la biomasa vegetal (materia orgánica que existe en un ecosistema forestal por encima y por debajo del suelo). Cualquier actividad que tenga un efecto positivo sobre la capacidad de un área dada para almacenar y capturar carbono, podría ser considerada potencialmente como una opción para reducir $\mathrm{CO}_{2}$ de la atmósfera. Se han identificado dos estrategias principales de manejo del carbono: 1) Incrementar la cantidad o tasa de acumulación del carbono al crear o incrementar sumideros de carbono y, 2) prevenir o reducir la tasa de liberación del carbono ya fijado en los sumideros existentes (Jiménez, 2005).

La captura de carbono permitirá, a largo plazo, contribuir directamente en la mitigación del cambio climático (Ordóñez et al., 2001). La determinación adecuada de la biomasa de un bosque es un elemento de gran importancia, debido a que ésta permite determinar también los montos de carbono y otros elementos químicos existentes en cada uno de sus componentes (Brown et al., 1996).

Para la estimación de la biomasa se emplean diferentes métodos de cálculo, entre los que destacan los basados en ecuaciones matemáticas y aquellos para los que se generan factores de expansión. El método que utiliza factores de expansión se aplica cuando no existe la información detallada de un inventario forestal con los parámetros de cada árbol individual. Este consiste en multiplicar la biomasa de los fustes por el factor de expansión de biomasa, dando como 
resultado el valor de biomasa aérea total. La biomasa de los fustes es el producto de su volumen (volumen comercial) por la densidad básica promedio de las especies en cuestión (Brown et al., 1989).

El uso de peso para expresar la cantidad de productos forestales y la necesidad actual de medir la biomasa de rodales, ha impulsado el desarrollo de métodos para estimar el peso de los árboles en pie (Husch, 2001). Las relaciones entre las dimensiones del fuste $y$ la cantidad de biomasa se han empleado para estimar la biomasa (Bartelink, 1996; González, 2001) y el carbono presente en diferentes tipos de vegetación (Brown et al., 1989; De Jong et al., 1995).

\section{OBJETIVO}

El objetivo del presente trabajo fue la estimación de la biomasa y el contenido de carbono capturado en bosques de Pinus cooperi Blanco, en Durango, México.

\section{METODOLOGÍA}

El presente estudio se realizó en el ejido La Victoria, ubicado al Sudoeste del estado de Durango, en el municipio de Pueblo Nuevo. Está enmarcado geográficamente entre los paralelos $23^{\circ} 40^{\prime} 04^{\prime \prime}$ y $23^{\circ} 47^{\prime} 54^{\prime \prime}$ de latitud Norte y los meridianos $105^{\circ} 21^{\prime} 31^{\prime \prime}$ y $105^{\circ} 29^{\prime} 52^{\prime \prime}$ de longitud Oeste.

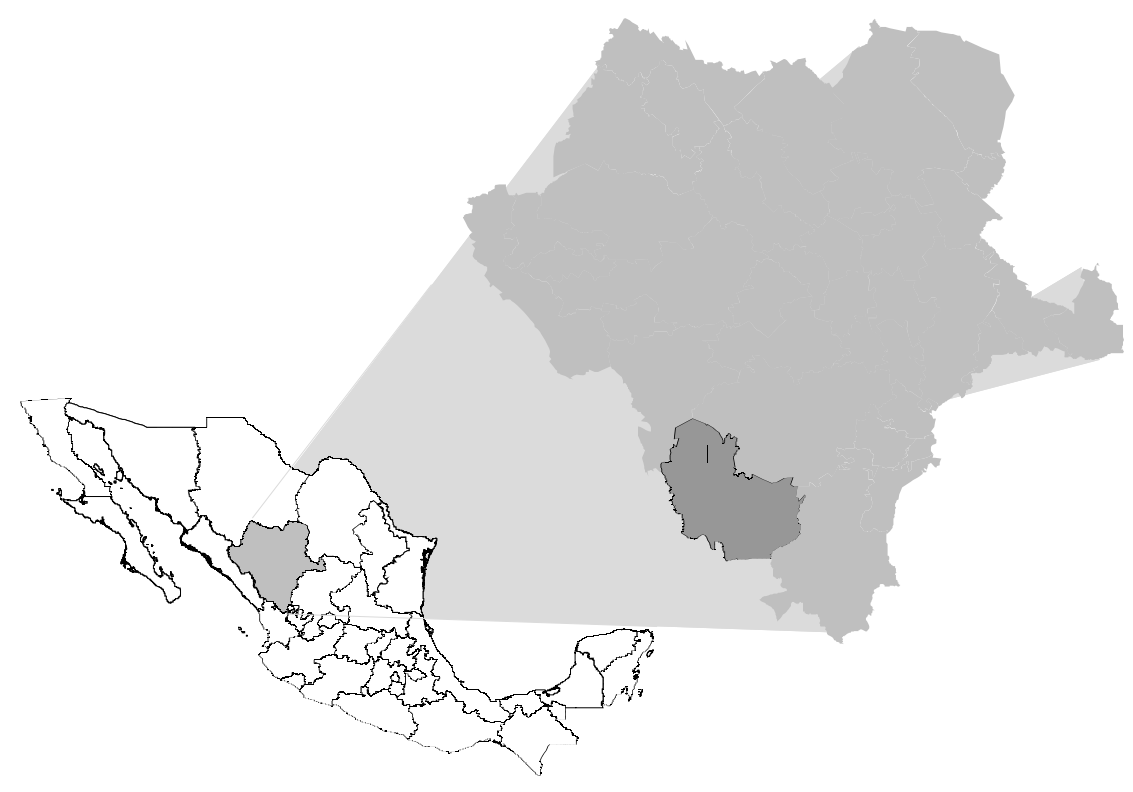

Figura 1. Localización del área de investigación. 
Orográficamente, el ejido La Victoria se encuentra ubicado en la provincia fisiográfica de la Sierra Madre Occidental, específicamente en la subprovincia de Gran Meseta y Cañones Duranguenses y Mesetas y Cañadas del Sur. El tipo de roca predominante es ígnea extrusiva ácida. De acuerdo con la carta edafológica del INEGI (1984), los suelos del predio son de tipo cambisol, regosol y litosol con textura predominantemente gruesa a media. La vegetación en la mayor parte del predio está formada por bosques de pino-encino, con distintas condiciones de productividad. Las principales especies de pino son Pinus cooperi Blanco, $P$. durangensis Martínez y $P$. leiophylla Schl. et Cham.

Los datos dasométricos para el estudio provinieron de ocho parcelas permanentes de muestreo, rectangulares de $5000 \mathrm{~m}^{2}(100 \mathrm{~m} \times 50 \mathrm{~m}$, en total 4 hectáreas), en donde se presenta principalmente Pinus cooperi Blanco. La base de datos la constituyeron 633 árboles muestreados a los que se les midió el diámetro normal y la altura total. Para la estimación del volumen se empleó la siguiente ecuación volumétrica $V=$ $0,0548+0,424^{*} D^{2} \mathrm{H}$ (Hernández y Galván, 2003), cuyo coeficiente de determinación es 0,92 . Para obtener la biomasa del fuste se multiplicó el volumen total por la densidad de Pinus cooperi, la cual es de $0,43 \mathrm{gr} / \mathrm{cm}^{3}$ (Nájera, 2005). Una vez determinados los valores de biomasa se procedió a estimar los valores de carbono, multiplicando la biomasa por la concentración de carbono promedio para coníferas 0,50 (valor citado en los inventarios de gases de efecto invernadero, sector forestal para México, IPCC, 2001).

A partir de datos de inventarios forestales, se empleó el método propuesto por Brown et al. (1989), el cual consiste en ecuaciones matemáticas, ya que se cuenta con los datos básicos (DAP, altura total y densidad básica de la madera), para aplicar la ecuación de estimación de biomasa más precisa. Los modelos empleados en este estudio (Tabla 1) para la estimación de la biomasa son los propuestos por Sanquetta et al. (2002).

Tabla 1. Modelos para la estimación de la biomasa.

\begin{tabular}{ll}
\hline Modelo & Ecuación \\
\hline 1 & $\mathrm{~B}=\mathrm{a}+\mathrm{bD}+\mathrm{c}\left(\mathrm{D}^{2}+\mathrm{H}\right)$ \\
2 & $\mathrm{~B}=\mathrm{a}+\mathrm{bD}+\mathrm{c} \mathrm{D}^{2}+\mathrm{d}\left(\mathrm{D}^{2} \mathrm{H}\right)$ \\
3 & $\mathrm{~B}=\mathrm{a}+\mathrm{b} \mathrm{D}^{2}+\mathrm{c}\left(\mathrm{D}^{2} \mathrm{H}\right)$ \\
4 & $\mathrm{~B}=\mathrm{a}+\mathrm{bD}+\mathrm{cH}$ \\
5 & $\mathrm{~B}=\mathrm{a} \mathrm{D}^{\mathrm{b}+\mathrm{H}^{\mathrm{c}}}$ \\
\hline
\end{tabular}

Donde:

$\mathrm{B}=$ biomasa, $\mathrm{D}=$ diámetro normal $(1,30 \mathrm{~m}), \mathrm{H}=$ altura total $(\mathrm{m}), \mathrm{a}, \mathrm{b}, \mathrm{c}, \mathrm{d}=$ parámetros estadísticos.

Se desarrolló el procesamiento matemático de la información dasométrica, seleccionando las mejores funciones de biomasa y de carbono capturado, ubicando los individuos en rangos de categorías diamétricas de 5 centímetros. Una vez determinado el carbono contenido y considerando los modelos matemáticos de biomasa y carbono ajustados, se procedió a estimar este parámetro para las diferentes categorías diamétricas y de altura. Posteriormente, con los modelos seleccionados, se elaboraron tablas similares a las de volumen, que incorporan por cada categoría diamétrica y de altura los valores de biomasa y carbono contenido estimado, respectivamente. 
El procesamiento de los datos se realizó en el paquete estadístico STATISTICA $7.0^{\circledR}$. En la evaluación de la calidad de los ajustes se analizaron los coeficientes de determinación $\left(R^{2}\right)$ y el error estándar porcentual $\left(\mathrm{S}_{\mathrm{yx}} \%\right)$, seleccionando aquel modelo que presentó menor error estándar porcentual y mayor valor del coeficiente de determinación.

\section{RESULTADOS Y DISCUSIÓN}

En las figuras 2 y 3 se observa de manera gráfica la tendencia ascendente en la producción de biomasa y del carbono contenido, de acuerdo a las categorías diamétricas de los árboles objeto de estudio.

Las tablas 2 y 3 muestran los coeficientes de regresión de los modelos de biomasa y carbono capturado ajustados, así como los respectivos coeficientes de determinación y los errores estándar porcentuales, que fueron los criterios de selección utilizados.
Puede observarse que tres modelos presentan un $R^{2}$ similar y el error estándar porcentual con poca variación. Para la estimación de la biomasa pueden utilizarse, por tanto, los modelos 1, 2 y 3, ya que presentan muy buenos ajustes. Para este estudio se seleccionó el modelo 2 (Figura 4), que presentó el mejor ajuste con un $\mathrm{S}_{\mathrm{x}} \%$ de 11,69 y un $\mathrm{R}^{2}$ de 0,96 , cumpliendo con el criterio de selección antes mencionado.

Para el caso del carbono contenido se tienen cuatro modelos que presentan un buen ajuste con un $\mathrm{R}^{2}$ de 0,99 y error estándar porcentual que va de 2,46 a 4,90\% (Tabla 3). El modelo seleccionado para la estimación del carbono contenido fue el dos con un $S_{x} \%$ de 2,46 y un $R^{2}$ de 0,99 (Figura 5). En general, considerando las variables analizadas, los modelos presentaron un comportamiento muy semejante en términos de calidad de ajuste a los datos.

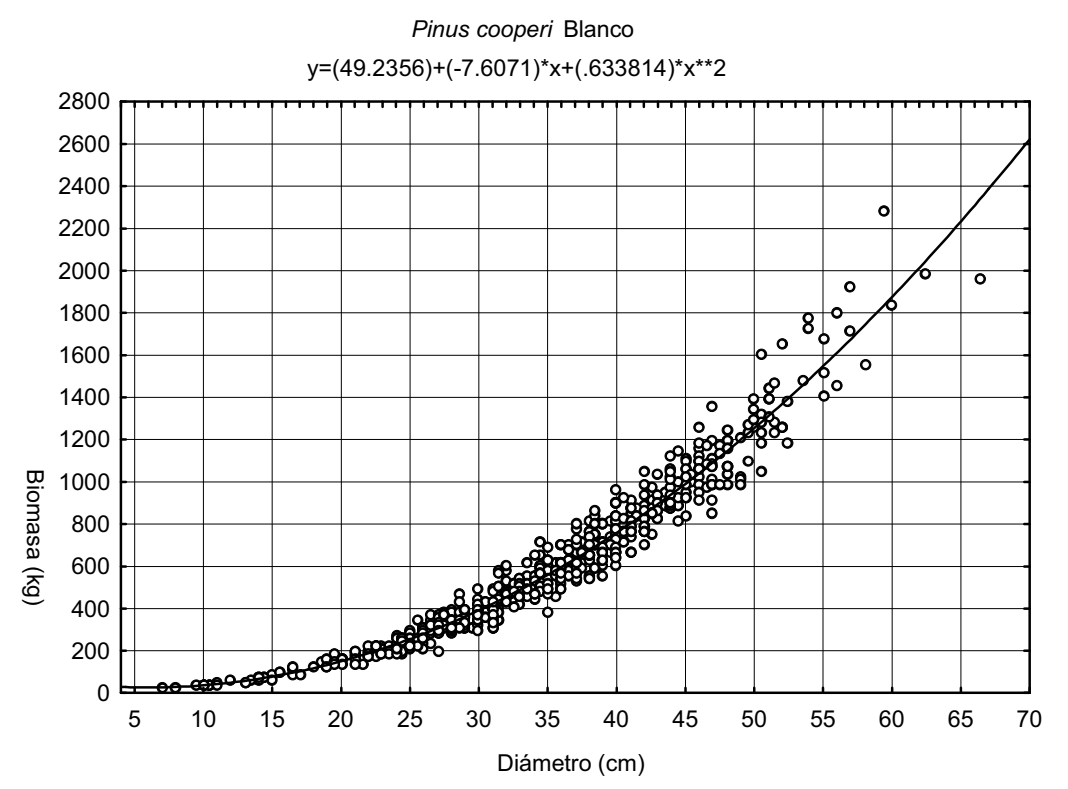

Figura 2. Relación entre biomasa y diámetro normal 


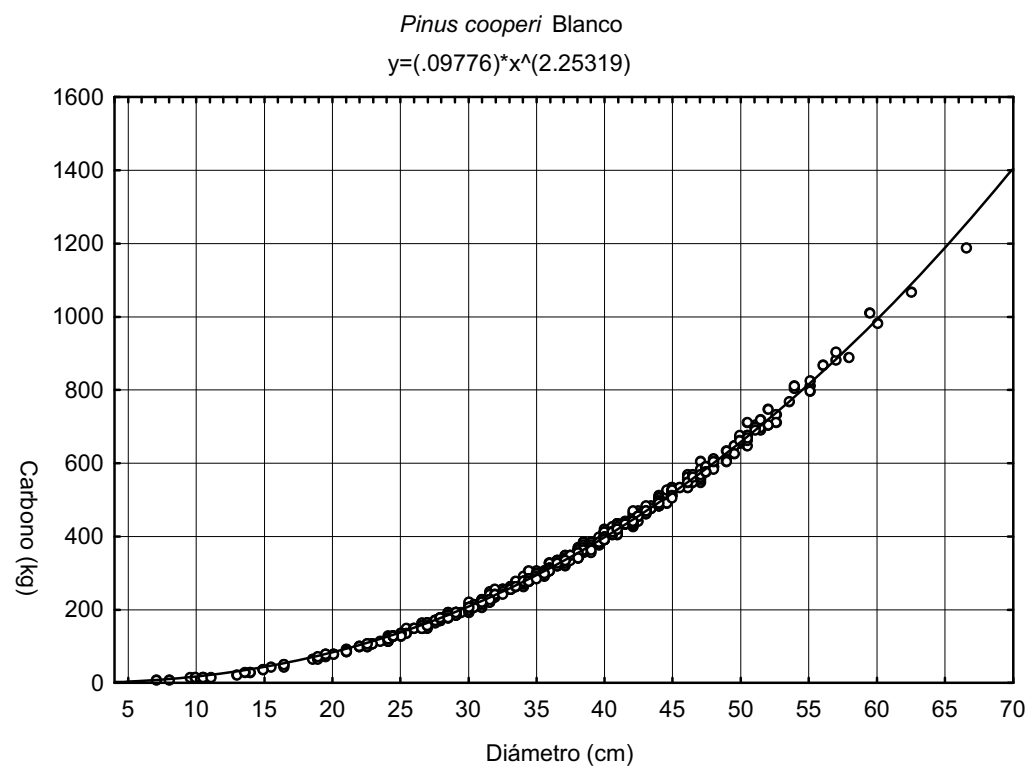

Figura 3. Relación entre carbono contenido y diámetro normal

Modelo 2: $\mathrm{B}=\mathrm{a} 0+\mathrm{a} 1^{*} \mathrm{~d}+\mathrm{a} 2^{*} \mathrm{~d}^{* *} 2+\mathrm{a} 3^{*}\left(\mathrm{~d}^{* *} 2^{*} \mathrm{~h}\right)$ $B=(22.3476)+(-4.497)^{*} x+(.491071)^{*} x^{* \star} 2+(.003859)^{*}\left(x^{* \star} 2^{*} y\right)$

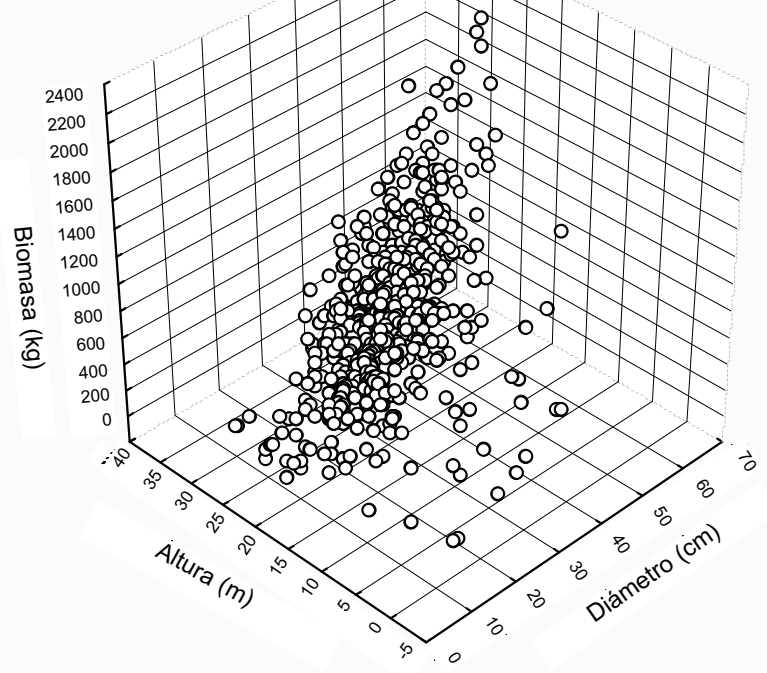

Figura 4. Gráfica del modelo ajustado para la estimación de biomasa seca 
Tabla 2. Parámetros de ajuste para biomasa de Pinus cooperi Blanco

\begin{tabular}{|c|c|c|c|c|c|c|}
\hline Modelos & $\mathbf{a}$ & b & c & d & $\mathbf{R}^{2}$ & $S_{y y} \%$ \\
\hline (1) $B=a+b D+c\left(D^{2}+H\right)$ & 41,9834 & $-7,9540$ & 0,6373 & & 0,96 & 12,15 \\
\hline 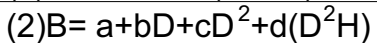 & 22,3476 & $-4,9470$ & 0,4911 & 0,0039 & 0,96 & 11,69 \\
\hline (3) $\mathrm{B}=\mathrm{a}+\mathrm{b} \mathrm{D}^{2}+c\left(\mathrm{D}^{2} \mathrm{H}\right)$ & $-57,6570$ & 0,4119 & 0,0042 & & 0,96 & 11,79 \\
\hline (4) $\mathrm{B}=\mathrm{a}+\mathrm{bD}+\mathrm{cH}$ & $-733,870$ & 34,6275 & 6,0467 & & 0,90 & 18,20 \\
\hline$(5) B=a D^{b}+H^{c}$ & 0,1428 & 2,2107 & 0,1326 & & 0,95 & 12,55 \\
\hline
\end{tabular}

Tabla 3. Parámetros de ajuste para carbono capturado en Pinus cooperi Blanco

\begin{tabular}{|l|r|r|r|c|r|r|}
\hline \multicolumn{1}{|c|}{ Modelos } & \multicolumn{1}{c|}{ a } & \multicolumn{1}{c|}{$\mathbf{b}$} & \multicolumn{1}{c|}{$\mathbf{c}$} & \multicolumn{1}{c|}{$\mathbf{d}$} & \multicolumn{1}{c|}{$\mathbf{R}^{\mathbf{2}}$} & $\mathbf{S}_{\mathbf{y x}} \%$ \\
\hline$(1) \mathrm{B}=\mathrm{a}+\mathrm{bD}+\mathrm{c}\left(\mathrm{D}^{2}+\mathrm{H}\right)$ & 8,4937 & $-3,4482$ & 0,3254 & & 0,99 & 2,49 \\
\hline$(2) \mathrm{B}=\mathrm{a}+\mathrm{bD}+\mathrm{c} \mathrm{D}^{2}+\mathrm{d}\left(\mathrm{D}^{2} \mathrm{H}\right)$ & 11,5090 & $-3,1229$ & 0,3100 & 0,0004 & 0,99 & 2,46 \\
\hline$(3) \mathrm{B}=\mathrm{a}+\mathrm{b} \mathrm{D}^{2}+\mathrm{c}\left(\mathrm{D}^{2} \mathrm{H}\right)$ & $-38,9960$ & 0,2601 & 0,0006 & & 0,99 & 3,08 \\
\hline$(4) \mathrm{B}=\mathrm{a}+\mathrm{bD}+\mathrm{cH}$ & $-353,030$ & 18,6264 & 1,0868 & & 0,94 & 14,01 \\
\hline$(5) \mathrm{B}=\mathrm{a} \mathrm{D}^{\mathrm{b}}+\mathrm{H}^{\mathrm{c}}$ & 0,0897 & 2,2570 & 0,0220 & & 0,99 & 4,90 \\
\hline
\end{tabular}

Modelo 2: $\mathrm{Cc}=\mathrm{a} 0+\mathrm{a} 1^{*} \mathrm{~d}+\mathrm{a} 2^{*} \mathrm{~d}^{* *} 2+\mathrm{a} 3^{*}\left(\mathrm{~d}^{* *} 2^{*} \mathrm{~h}\right)$ $C_{c}=(11.509)+(-3.1229)^{*} x+(.310001)^{*} x^{* *} 2+(.426 e-3)^{*}\left(x^{\star *} 2^{*} y\right)$

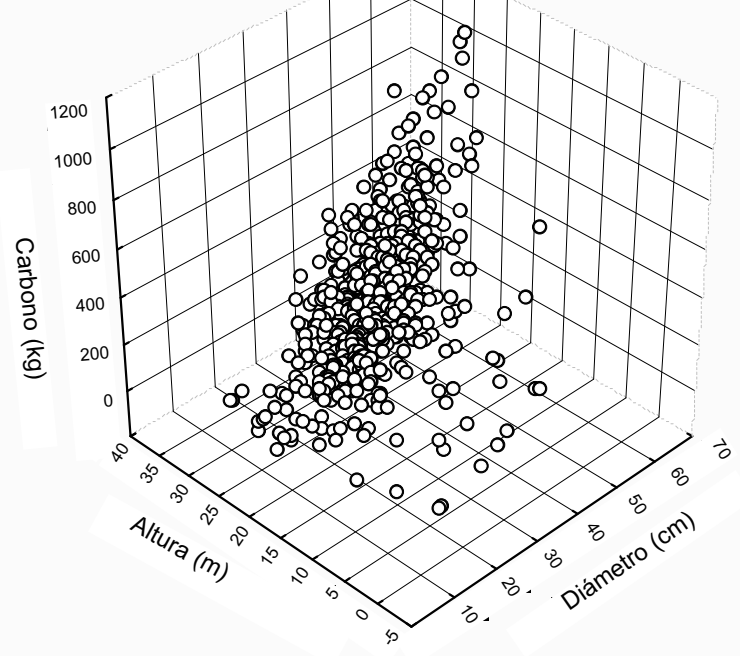

Figura 5. Gráfica del modelo ajustado para la estimación de carbono contenido 
Tabla 4. Biomasa estimada en peso seco y del carbono capturado en toneladas por hectárea para Pinus cooperi Blanco

\begin{tabular}{|c|c|c|c|c|c|}
\hline $\begin{array}{c}\text { Categoría } \\
\text { diamétrica } \\
\text { (cm) }\end{array}$ & Individuos & $\begin{array}{c}\text { Biomasa } \\
\text { estimada } \\
\text { Ton }\end{array}$ & $\begin{array}{c}\text { Carbono } \\
\text { contenido } \\
\text { Ton }\end{array}$ & $\begin{array}{c}\text { Biomasa } \\
\text { estimada } \\
\text { ton/ha }\end{array}$ & $\begin{array}{c}\text { Carbono } \\
\text { contenido } \\
\text { estimado } \\
\text { ton/ha }\end{array}$ \\
\hline 5 & 1 & 0,013 & 0,007 & 0,003 & 0,002 \\
\hline 10 & 12 & 0,318 & 0,166 & 0,079 & 0,041 \\
\hline 15 & 17 & 1,184 & 0,617 & 0,296 & 0,154 \\
\hline 20 & 26 & 3,943 & 2,054 & 0,986 & 0,514 \\
\hline 25 & 67 & 16,945 & 8,829 & 4,236 & 2,207 \\
\hline 30 & 124 & 47,988 & 25,002 & 11,997 & 6,250 \\
\hline 35 & 132 & 74,144 & 38,629 & 18,536 & 9,657 \\
\hline 40 & 119 & 90,291 & 47,042 & 22,573 & 11,760 \\
\hline 45 & 80 & 79,928 & 41,642 & 19,982 & 10,411 \\
\hline 50 & 38 & 48,364 & 25,198 & 12,091 & 6,299 \\
\hline 55 & 12 & 19,105 & 9,954 & 4,776 & 2,488 \\
\hline 60 & 3 & 5,685 & 2,962 & 1,421 & 0,740 \\
\hline 65 & 2 & 4,606 & 2,400 & 1,152 & 0,600 \\
\hline Total & $\mathbf{6 3 3}$ & $\mathbf{3 9 2 , 5 1 4}$ & $\mathbf{2 0 4 , 5 0 0}$ & $\mathbf{9 8 , 1 2 8}$ & $\mathbf{5 1 , 1 2 5}$ \\
\hline
\end{tabular}

En la tabla 4 se presentan los individuos evaluados por categoría diamétrica, en las ocho parcelas muestreadas, incluyendo la biomasa estimada en peso seco y el carbono contenido en toneladas por hectárea.

El valor promedio por hectárea estimado, para fuste limpio $(51,12$ ton/ha), es mayor al obtenido por Domínguez et al. (2007) en bosques mixtos de Pinus teocote y Pinus pseudostrobus en el Sur de Nuevo León $(45,18$ ton/ha).
En las tablas 5 y 6 se presentan los valores de biomasa y carbono contenido por categoría diamétrica y de altura. A partir de estos resultados se puede estimar la cantidad de biomasa y carbono almacenado en los individuos de diferentes categorías diamétricas y de altura, y constituyen valores de referencia para estimar la acumulación promedio por unidad de superficie de bosques de Pinus cooperi Blanco, a partir de distribuciones diamétricas. 
ลิ

N

กั

8
$\frac{0}{\pi}$
$\frac{\pi}{0}$
0
8
8
8
0
0

i

몬

은

$\stackrel{0}{2}$

8

ర্

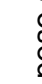

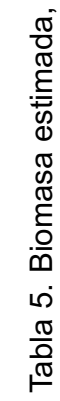

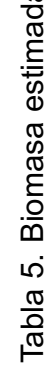

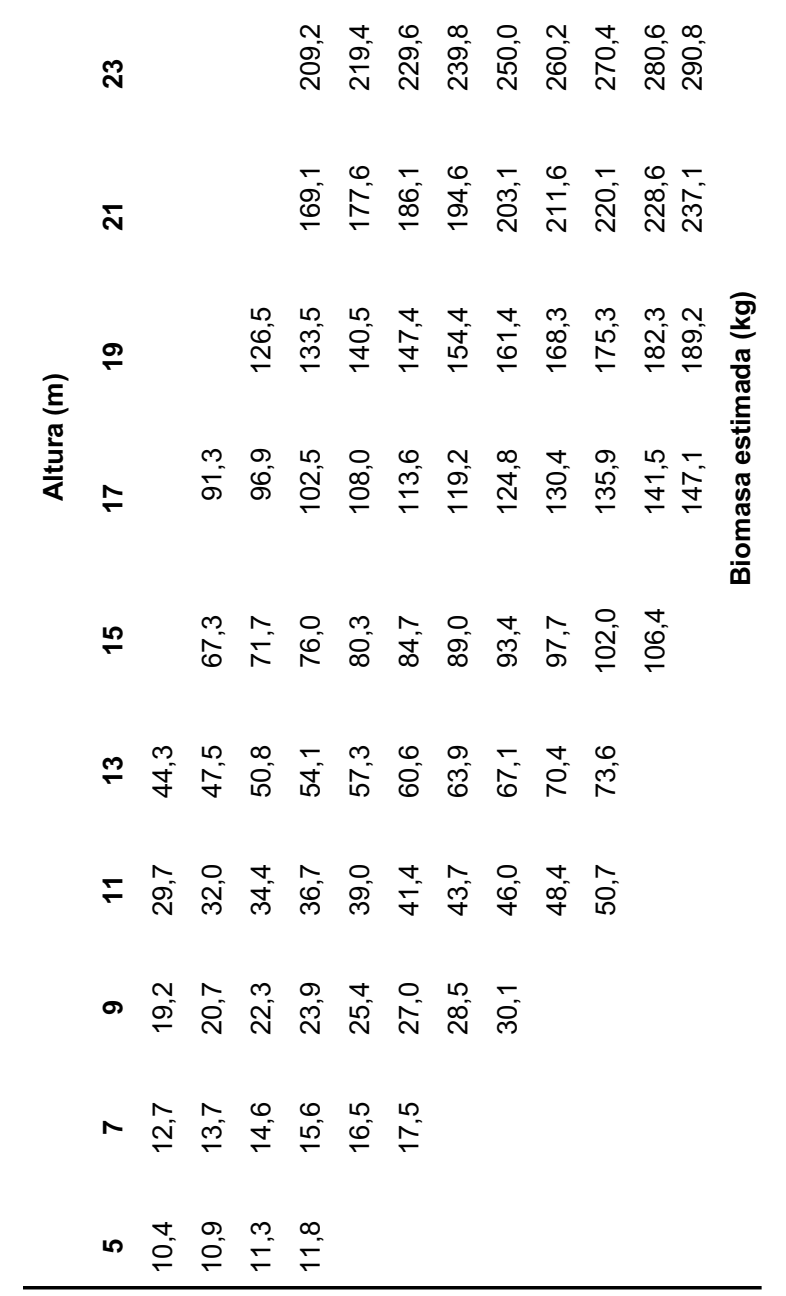

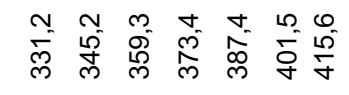

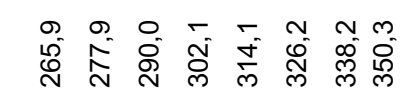




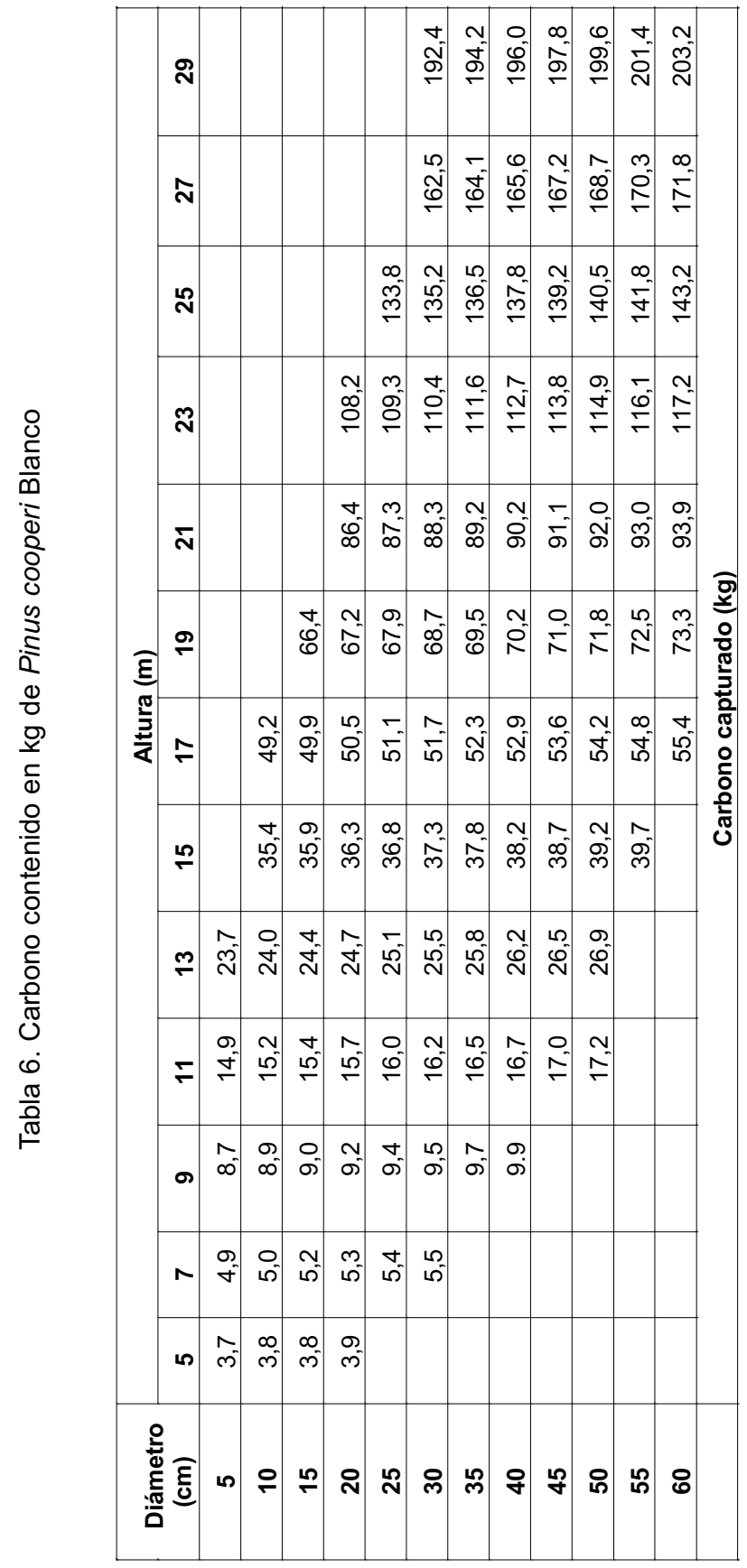




\section{CONCLUSIONES}

Mediante la aplicación de ecuaciones alométricas de biomasa es posible estimar el carbono contenido.

Los modelos evaluados presentaron un comportamiento muy semejante en términos de calidad de ajuste a los datos y variables analizadas. Las estimaciones se pueden realizar con cualquiera de las cuatro ecuaciones utilizadas, ya que presentan un error estándar porcentual bajo y un coeficiente de determinación muy similar.

Las tablas desarrolladas para evaluar la cantidad de biomasa y de carbono contenido en rodales de Pinus cooperi Blanco, permiten hacer una rápida estimación del carbono almacenado en estos ecosistemas, considerando las variables diámetro y altura.

Las tablas de estimación de biomasa y carbono desarrolladas en este trabajo permiten contar con valores de referencia para bosques de Pinus cooperi Blanco, en el secuestro de $\mathrm{CO}_{2}$.

\section{RECONOCIMIENTO}

Al Consejo Nacional de Ciencia y Tecnología (CONACyT), por el financiamiento otorgado al Proyecto 41181-Z.

\section{REFERENCIAS}

Bartelink, H. H. 1996. Allometric relationships on biomass and needle area of Douglas-fir. Forest Ecology and Management 86:193-203.

Brown, S., J. R. Gillespe A. and A. E. Lugo. 1989. Biomass estimation for tropical forest with applications to forest inventory data. Forest Science 35(4): 881-902.
Brown, S., J. Sathaye, M. Cannell y P. Kauppi. 1996. Mitigation of carbon emission to the atmosphere by forest management. Commonwealth Forestry Review, 75(1): 80-91.

De Jong, B. H. J., G. Montoya G., K. Nelson, L. Soto P. y R. Tipper. 1995. Community forest management and carbon sequestration. A Feasibility study from Chiapas, México. Interciencia 20(6):409-416.

Centro de Investigación de la Universidad del Pacífico. 2001. Boletín del área de recursos naturales y del medio ambiente,11(10). Octubre, 2001.

Domínguez C. G., O. A. Aguirre C., J. Jiménez P. y H. Villalón M. 2007. Evaluación del contenido de carbono en bosques del Sur de Nuevo León. En preparación.

Gasparri, I. y E. Manghi. 2004. Estimación de volumen, biomasa y contenido de carbono de las regiones forestales argentinas. Dirección de Bosques, Secretaría de Ambiente y Desarrollo Sustentable. Unidad de Manejo del Sistema de Evaluación Forestal. Argentina. 26 pp.

González B. N. 2001. Ajuste y validación de modelos para estimar biomasa y rendimiento $e$ incremento en biomasa de plantaciones forestales del estado de Durango, México. Tesis de Maestría. Facultad de Ciencias Forestales. UANL. 76 pp.

Hernández F., J. y J. D. Galván M., 2003. Ecuaciones para estimar volumen total y comercial de Pinus cooperi del ejido La Victoria de la región de Pueblo Nuevo, Durango. VI Congreso Mexicano de Recursos Forestales. Sociedad Mexicana de Recursos Forestales. Universidad 
Autónoma de San Luis Potosí. Facultad de Agronomía. pp. 37-38.

Husch, B. 2001. Estimación del contenido de carbono de los bosques. Simposio Internacional Medición y Monitoreo de la Captura de Carbono en Ecosistemas Forestales. Valdivia, Chile.

INEGI. 1984. Carta topográfica. Escala 1:50,000. El Salto, Durango. F13A18 spp. México.

INTERGOVERNMENTAL PANEL ON CLIMATE CHANGE (IPCC). 2001. Third assessment report-climate change, The scientific basis: summary for policymakers. A report of working group I of the Intergovernmental Panel on Climate Change. UNEP-WMO. P. 7. www.ipcc.ch.

Jiménez P., J. 2005. Fijación de carbono en la Reserva de la Biosfera "E Cielo". Informe Técnico Final. Facultad de Ciencias Forestales. UANL. México.

Karjalainen, T. 1996. Dynamics and potentials of carbon sequestration in managed stands and wood products under changing climatic conditions. Forest Ecology and Management 80:113-132.

Masera R., O. 2001. Carbon sequestration dynamics in forestry projects: The CO2FIX V.2. Model approach. Instituto de Ecología, UNAM. $13 p$.

Nájera L., J.A. 2005. Caracterización de maderas de la región de El Salto, Duango. Instituto Tecnológico Forestal. Informe de Proyecto.
Ordóñez, J. A. B. 1999. Captura de carbono en un bosque templado: El caso de San Juan Nuevo, Michoacán. Instituto Nacional de Ecología, SEMARNAP, México, D.F. Junio. $72 \mathrm{p}$.

Ordóñez, J. A. B., H. J. de Jong y O. Masera. 2001. Almacenamiento de carbono en un bosque de Pinus pseudostrobus en Nuevo San Juan, Michoacán. Madera y Bosques 7(2): 27-47.

Ordóñez, J. A. B. y O. Masera. 2001. Captura de carbono ante el cambio climático. Madera y Bosques 7 (1): 312.

Sanquetta C., R., L. Farinha W. y J.E. Arce. 2002. Ecuaciones de biomasa aérea y subterránea en plantaciones de Pinus taeda en el sur del Estado de Paraná, Brasil. Patagónica Forestal. Revista forestal del sur argentino. $13 \mathrm{p}$.

SEMARNAT. 2001. México $2^{a}$ Comunicación Nacional ante la Convención Marco de las Naciones Unidad sobre el Cambio Climático. Comité Intersecretarial sobre Cambio Climático. Secretaría de Medio Ambiente y Recursos Naturales (SEMARNAT), Instituto Nacional de Ecología (INE). 374 pp.

Schlegel, B. 2001. Estimación de biomasa y carbono en bosques del tipo forestal siempre verde. Universidad Austral de Chile. Simposio Internacional Medición y Monitoreo de la Captura de Carbono en Ecosistemas Forestales. Valdivia, Chile. 13 p.

Manuscrito recibido el 11 de enero de 2006.

Aceptado el 21 de agosto de 2006.

Este documento debe citarse como: Pimienta De la Torre, D. J., G. Domínguez Cabrera, O. Aguirre Calderón, F. Javier Hernández y J. Jiménez Pérez. Estimación de biomasa y contenido de carbono de Pinus cooperi Blanco, en Pueblo Nuevo, Durango. Madera y Bosques 13(1):35-46. 